\title{
On the ionospheric coupling of auroral electric fields
}

\author{
G. T. Marklund \\ Space and Plasma Physics, School of Electrical Engineering, Royal Institute of Technology, KTH 10044 Stockholm, Sweden
}

Received: 22 December 2008 - Revised: 9 April 2009 - Accepted: 20 April 2009 - Published: 30 April 2009

\begin{abstract}
The quasi-static coupling of high-altitude potential structures and electric fields to the ionosphere is discussed with particular focus on the downward field-aligned current (FAC) region. Results are presented from a preliminary analysis of a selection of electric field events observed by Cluster above the acceleration region. The degree of coupling is here estimated as the ratio between the magnetic field-aligned potential drop, $\Delta \Phi_{\mathrm{II}}$, as inferred from the characteristic energy of upward ion (electron) beams for the upward (downward) current region and the high-altitude perpendicular (to $\mathbf{B}$ ) potential, $\Delta \Phi_{\perp}$, as calculated by integrating the perpendicular electric field across the structure. For upward currents, the coupling can be expressed analytically, using the linear current-voltage relation, as outlined by Weimer et al. (1985). This gives a scale size dependent coupling where structures are coupled (decoupled) above (below) a critical scale size. For downward currents, the currentvoltage relation is highly non-linear which complicates the understanding of how the coupling works. Results from this experimental study indicate that small-scale structures are decoupled, similar to small-scale structures in the upward current region. There are, however, exceptions to this rule as illustrated by Cluster results of small-scale intense electric fields, correlated with downward currents, indicating a perfect coupling between the ionosphere and Cluster altitude.
\end{abstract}

\section{Introduction}

A key issue in magnetospheric and auroral physics is how electrons and ions, producing discrete aurora and contributing to planetary plasma escape, gain their energy. Particle acceleration is a key issue for a vast number of phenomena in space and astrophysical plasmas. It was the main scientific

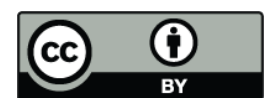

Correspondence to: G. T. Marklund (goran.marklund@ee.kth.se) objective for the Viking and Freja satellite missions which made many pioneering contributions on this topic (Marklund et al., 2004). During auroral activity, quasi-static electric potential structures are formed in the transition region between the ionosphere and the magnetosphere. In the upward current region, negative potential structures associated with converging electric fields and an upward magnetic-fieldaligned component, are formed at altitudes between 1 and 2 $R_{E}$. When passing through such potential drops, the Earthward moving electrons will increase their energy, resulting in a penetration deeper into the atmosphere and an intensification of the aurora. In the downward current region, potential structures of opposite polarity, with diverging electric fields and a downward pointing electric field, are formed at altitudes between 1000 and $4000 \mathrm{~km}$, accelerating electrons away from Earth. Characteristics of the downward current region and the duality between the two opposite auroral current branches, were discussed by Marklund et al. (1994, 1997) using Freja observations and by Marklund et al. (2001, 2004, 2006) using Cluster observations. FAST satellite data have added much new insight into the physics of the aurora, including the downward current region (Carlson et al., 1998; Ergun et al., 1998, 2001; Lynch et al., 2002). In addition to quasi-static acceleration, acceleration by inertial or kinetic Alfvén waves, play a crucial and sometimes dominant role for certain types of aurora (Chaston et al., 2003). Other types of wave particle interaction and turbulence occur in both the upward and downward current region, as discussed by Paschmann et al. (2003). In the downward current region, there are two kinds of turbulence known to be operative, a current-driven ion cyclotron turbulence and an electrostatic solitary wave turbulence (Jasperse et al., $2006 \mathrm{a}, \mathrm{b}$ ).

Weimer et al. (1985) studied the coupling of high-altitude electric fields to the ionosphere. They used a linear currentvoltage relation applicable to upward but not to downward currents, and derived a critical scale size, depending on the ratio a $/ \Sigma_{P}$. (where a is the field-aligned conductance and $\Sigma_{P}$ is the height-integrated Pedersen conductivity). For typical

Published by Copernicus Publications on behalf of the European Geosciences Union and the American Geophysical Union. 


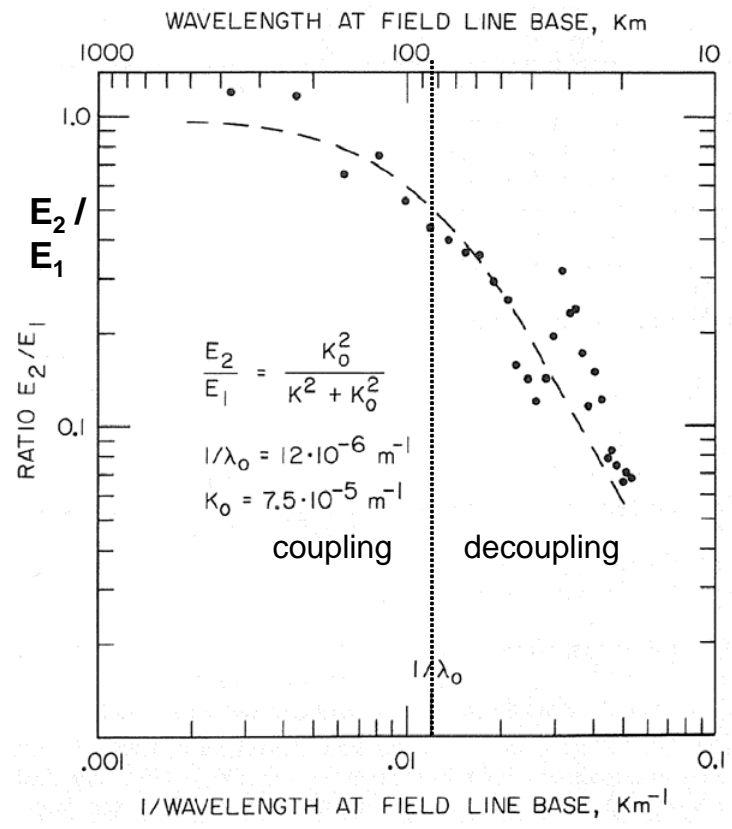

Fig. 1. Reproduction from Weimer et al. (1985) showing the ratio between the low-altitude electric field E2, measured by Dynamics Explorer-2 and the high-altitude electric field E1, measured by Dynamics Explorer 1, versus $1 / \lambda$ (wavelength or scale size) at the field line base.

parameter values, the critical scale size was found to be of the order of $100 \mathrm{~km}$. Larger (smaller) structures were found to be coupled to (decoupled from) the ionosphere, as illustrated in Fig. 1, showing the ratio between the low-altitude electric field E2, measured by Dynamics Explorer-2 and the highaltitude electric field E1, measured by Dynamics Explorer 1, versus the inverse of the wavelength (or scale size) at the field line base.

The coupling of electric fields in the downward current region depends critically on the current-voltage relation, which has been found to be highly non-linear, as shown in Fig. 2 from Elphic et al. (1998). A problem is that the depletion process limits the time that charge carriers are available in the original current channel. A way for the current sheet to adjust for this is to broaden, as has been observed for a few Cluster events (Marklund et al., 2001, 2006; Aikio et al., 2004) and in numerical simulations (Karlsson and Marklund, 1998; Streltsov and Marklund, 2006). The current-voltage relationship in the downward current region has been subject to several recent theoretical studies, a few of which are discussed below.

Temerin and Carlson (1998) used the continuity equation to determine how the density of the ionospheric electrons depends on the current and to find a potential that reduces the density of magnetospheric electrons by the same amount to keep the plasma quasi-neutral. The current-voltage relation was determined for simple profiles of the background

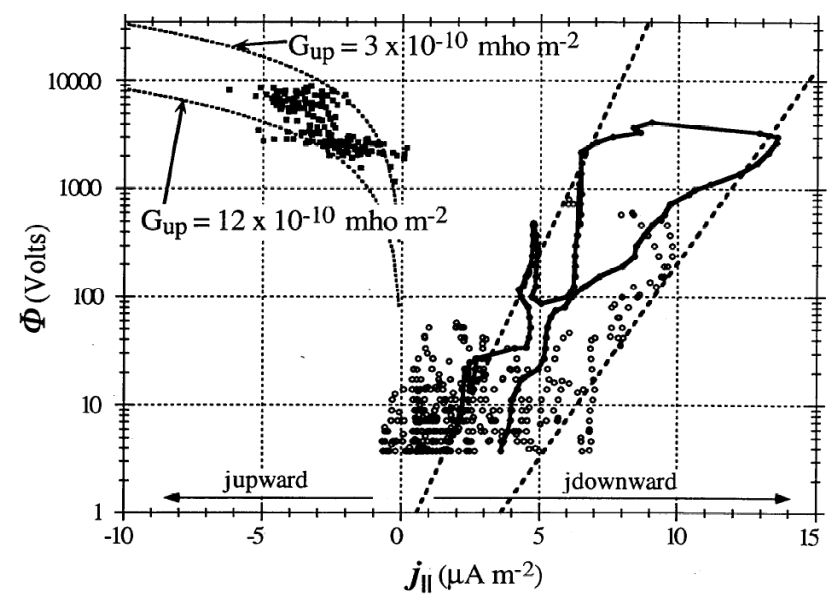

Fig. 2. Reproduction from Elphic et al. (1998) showing the relation between potentials and currents for different ranges of the magnetic field-aligned conductance.

ion density and the current found to be a few times larger in the downward current region compared to currents in the upward current region for similar potential drops. Potential drops up to a few thousand volts, as observed by FAST, are a necessary consequence of the observed current densities in the downward current region.

Using quasi-neutrality and steady-state plasma kinetic equations, Jasperse (1998) derived a model for the generation of a self-consistent downward pointing parallel electric field, an ion conic, and up-flowing field aligned electrons, all characteristic features of the downward field-aligned current region. Vedin and Rönnmark (2005) used the stationary Vlasov equation to derive the self-consistent potential in an auroral flux tube carrying downward current. The potential was found to peak at values of a few $\mathrm{kV}$, corresponding to altitudes around $1 R_{E}$ and downward FAC densities of $10 \mu \mathrm{A} / \mathrm{m}^{2}$. Below the peak there is a downward electric field and above the peak a weak upward electric field resulting in a very small potential difference between the ionosphere and the magnetosphere.

Hwang et al. (2006 a, b) used experimental data from the FAST satellite to estimate the coupling in the downward current region. They studied a large number of electric field events and found significant differences between sheet-like and curved structures, as illustrated in Fig. 3 for each of these structures. The panels show from top to bottom, time energy spectrogram for electrons, the axial electric field, the spin plane electric field, and the eastward and northward electric field components, the Poynting flux, and the perpendicular electric field (green line) together with the derivative of the characteristic electron energy (black line). For the sheet-like structure the Poynting flux is upward and the ratio between the electric field and the derivative of the characteristic electron energy (their coupling parameter), is 0.3 indicating a 


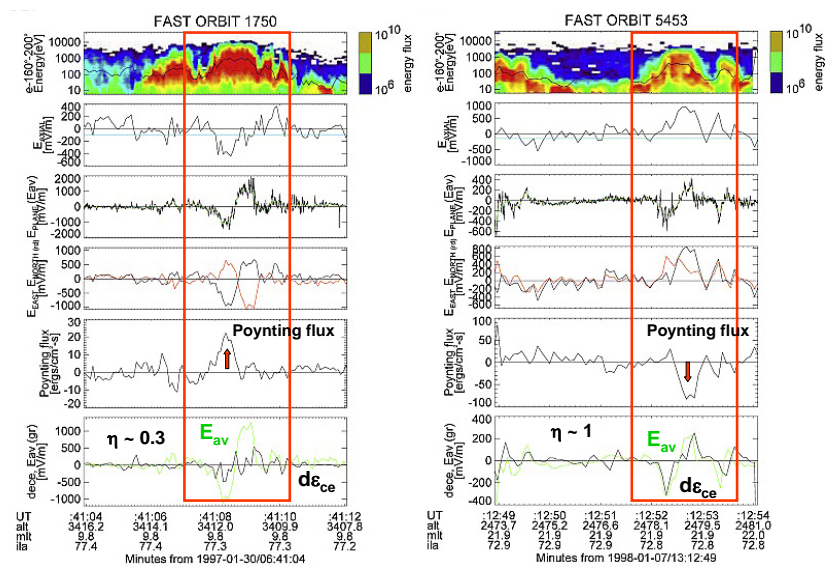

Fig. 3. Reproduction from Hwang et al. (2006) showing FAST data obtained for a sheet-like structure (left) and a curved structure (right). Panel 1 shows a time energy spectrogram for electrons. Panels 2-4 show the axial, the spin plane, and the eastward and northward electric field components, respectively. Panel 5 shows the Poynting flux and panel 6, the derivative of the characteristic electron energy (black line) and the perpendicular electric field (green line). Note that the ratio between these two parameters, the coupling ratio, is significantly less than one (around 0.3) for the sheet-like structure (left) and about 1 for the curved structure (right).

strong coupling. For the curved structure the results showed a downward directed Poynting flux and a coupling ratio of 1 , indicating a decoupling. From their dataset they concluded that potentials associated with curved structures, such as spirals and folds, had a tendency to be decoupled from the ionosphere $(0.5<\eta<1)$ whereas potentials associated with sheetlike structures, such as black arcs, were more coupled to the ionosphere $(0<\eta<0.5)$. The structures had typical scale sizes of a few ten $\mathrm{km}$, slightly larger than the scale sizes of 5$10 \mathrm{~km}$ derived from Cluster data.

Here, we shall apply a similar method to that used by Hwang et al. (2006a, b) to estimate the coupling of highlatitude electric fields to the ionosphere. Instead of FAST data we will use Cluster data collected high above the acceleration region as representative of the high-altitude electric field. The method is described in Sect. 2. The data were obtained by the electric field instrument (EFW, Gustafsson et al., 1997), the fluxgate magnetometer (FGM, Balogh et al., 1997), the electron instrument (PEACE, Johnstone et al., 1997) and the ion instrument (CIS, Reme et al., 1997). Section 3 presents and discusses the results for eight auroral events, six of which are from the downward current region, and two of which are from the upward current region. The events were selected using various selection criteria described below. It should be noted that the particle analyses in this study were performed by eye inspection rather than by using the particle distributions for calculating the characteristic energy of the ions and electrons. A more comprehensive study of a much larger number of Cluster events and using

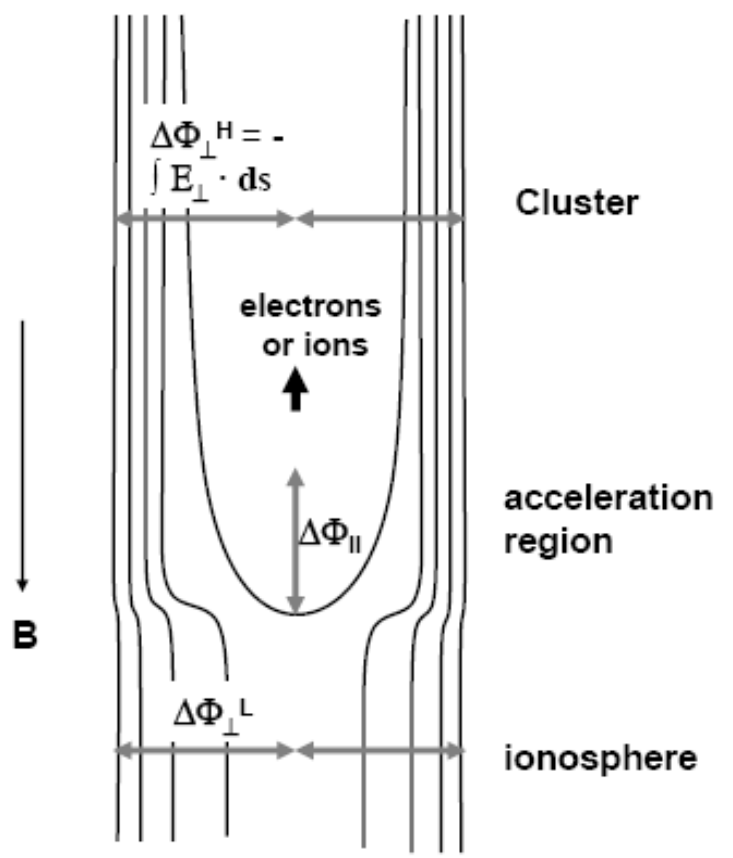

Fig. 4. Schematic illustrating the relationship between the high- and low-altitude auroral electric potential and between the parallel and the perpendicular (to $\mathbf{B}$ ) potential.

a more refined analysis of the particle data is planned for a forthcoming publication.

\section{Methodology}

The degree of coupling of auroral potentials is here calculated as the ratio between the parallel potential drop, $\Delta \Phi_{\text {II }}$ and the perpendicular potential, $\Delta \Phi_{\perp}$. The former is given by the characteristic energy of upward ion beams (in upward current regions), or upward electron beams (in downward current regions), and the latter by integrating the perpendicular electric field, $E_{\perp}$. The ratio $k=\Delta \Phi_{\mathrm{II}} / \Delta \Phi_{\perp}$ reveals whether the electric field couples completely $(k=0)$, is decoupled from $(k=1)$, or, partly couples $(0<k<1)$ to the ionosphere. The relationship between the high and low-altitude electric field and the potentials is illustrated in Fig. 4. (The coupling ratio used by Hwang et al. (2006 a, b) was the derivative of the characteristic energy $\left(e \Delta \Phi_{\mathrm{II}}\right)$ divided by the perpendicular electric field). The Cluster data were obtained well above the top of the acceleration region of the downward current region and typically above the acceleration region of the upward current region. The estimate of $\Delta \Phi_{\perp}$ refers to the time when Cluster crosses the structure (at $t=t_{0}$ ), whereas the $\Delta \Phi_{\text {II }}$ estimate refers to an earlier time $\left(t=t_{0}-\Delta t^{i}\right)$ when the ions (electrons) exited the top of the acceleration region. As an example, the times needed for up-going beams of $\mathrm{O}^{+}$ions to travel from the top of the acceleration region up to Cluster altitude, a 


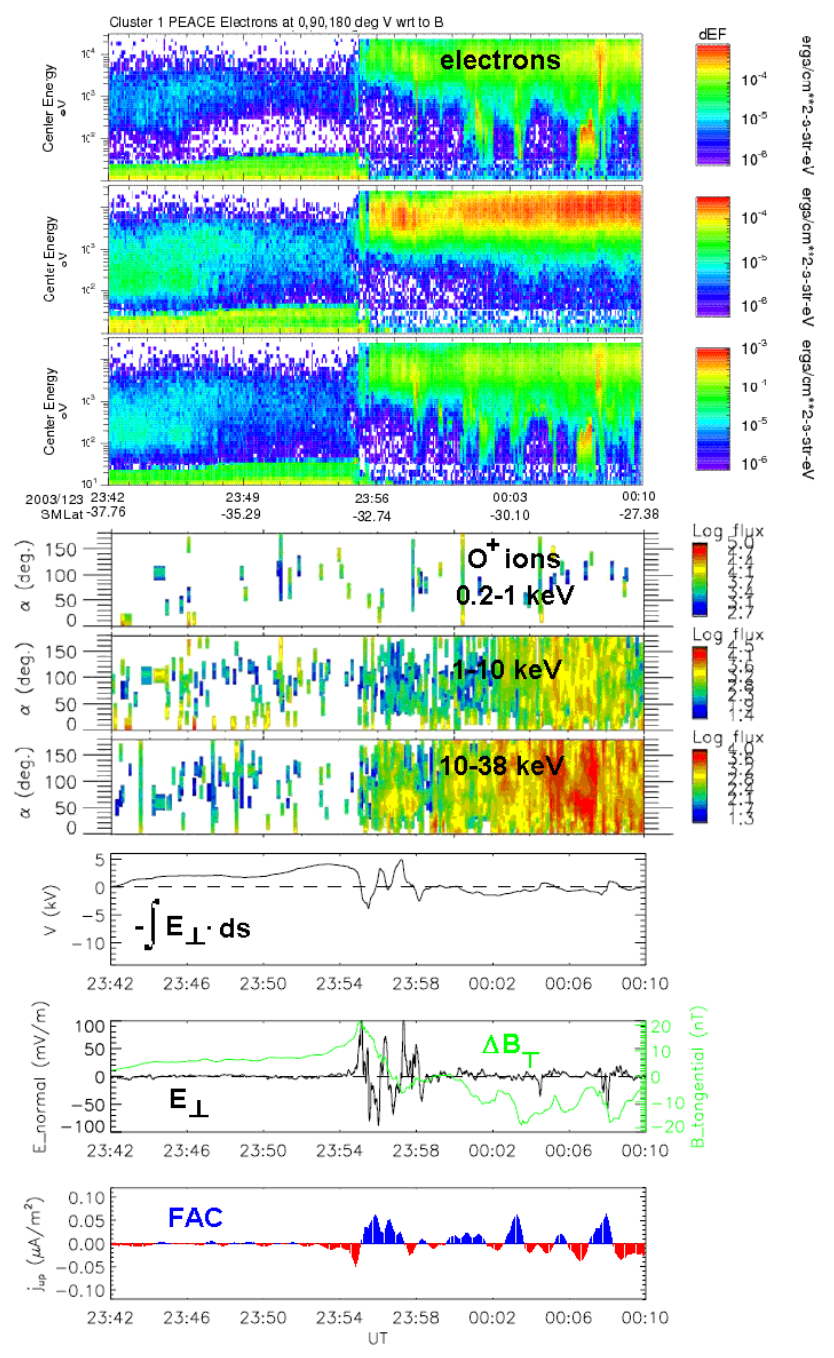

Fig. 5a. Observations from Cluster spacecraft 1, crossing an auroral plasma boundary in the southern hemisphere on 4 May 2003. The total time interval is $28 \mathrm{~min}$. Panels $1-3$ show time energy spectrograms of electrons, parallel, perpendicular and anti-parallel to the magnetic field, as measured by the PEACE instrument. Panels 4-6 show pitch-angle spectrograms versus time for $\mathrm{O}^{+}$ions at three energy ranges, $0.2-1 \mathrm{keV}, 1-10 \mathrm{keV}$, and $10-38 \mathrm{keV}$. Panels $7-$ 9 show the potential, the perpendicular electric field (black line) and the tangential residual magnetic field (green line) component, and field-aligned currents, in blue and red for upward and downward currents, respectively.

distance being a few $R_{E}$, are of the order of a few minutes. For electrons to travel the same distance the time is of the order of seconds. (The upward velocity derived from the parallel potential drop is likely to be an overestimate, since the derivation assumes that the upward beams are strictlyfield-aligned, the degree of which can be checked for each particular event. To obtain a more exact value of the travel times from the Cluster data requires a much more rigorous analysis of the data which is beyond the scope of this study, aimed at illustrating the general methodology). Another uncertainty is the finite crossing time of the structures. The k-value is therefore representative only on a time scale comparable to or larger than the crossing and transport times.

\section{Selection criteria}

1. EFW, FGM, CIS, PEACE data should be available and of high quality.

2. The angle between the magnetic field direction and the spin plane should exceed 5 degrees (for accurate values of all three electric field components).

3. The events should be limited to auroral crossings at geocentric distances between 4 and 7 Earth radii (at higher altitudes, temporal effects due to, for example inductive electric fields will increase the uncertainty of the analysis).

4. The events should be of quasi-static rather than temporal nature, which should be possible to determine using the method by Karlsson et al. (2004), applying cross-correlation and minimum-B variance analysis to the Cluster data.

5. The time intervals between the spacecraft should preferably range between $10 s<\delta T<20 \mathrm{~min}$, and the number of $s / c$ with good data should be at least 3, to enable monitoring of the evolution.

In addition, the database should preferably include electric field structures covering a broad range of spatial scales, from $1 \mathrm{~km}$ up to $100-200 \mathrm{~km}$, mapped to the ionosphere, and will be used for a more extensive study on this topic.

\section{Results and discussion}

Figure $5 \mathrm{a}$ and $\mathrm{b}$ are shown to illustrate the general methodology, where Cluster observations have been used to estimate the parallel and perpendicular potentials for a quasi-static potential structure in the upward current region. Figure 5a, panels 1-3, shows time energy spectrograms of electrons, parallel, perpendicular, and anti-parallel to the magnetic field, measured by the PEACE instrument. Panels 4-6 show pitchangle spectrograms versus time for oxygen ions at three energy ranges, $0.2-1 \mathrm{keV}, 1-10 \mathrm{keV}$, and $10-38 \mathrm{keV}$. Panels $7-$ 9 show the potential, the perpendicular electric field (green line) and tangential residual magnetic field component, and field-aligned currents, in blue and red for upward and downward currents, respectively. The region of upward FAC is associated with intense converging electric field structures, a high-altitude signature of the primary acceleration region. The top panel of Fig. 5b shows the parallel or acceleration potential inferred from the characteristic energy of the upward ion beams. The two curves labeled $22^{\circ}$ and $90^{\circ}$ in the 


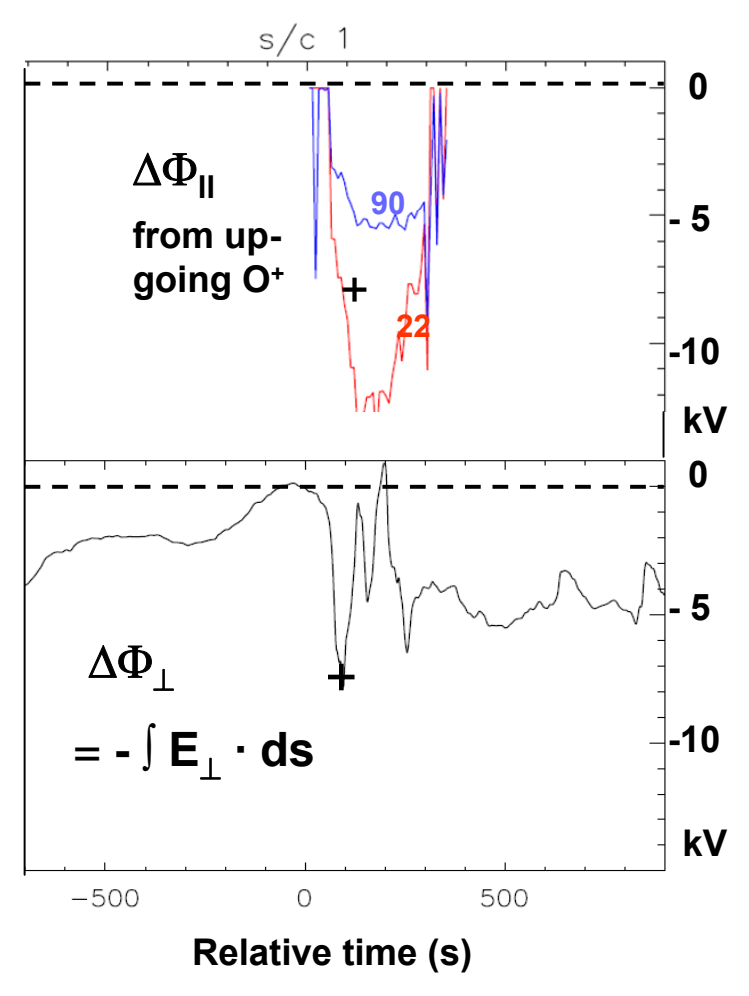

Fig. 5b. Comparison of inferred parallel potential drop, $\Delta \Phi_{\mathrm{II}}$, and perpendicular potential, $\Delta \Phi_{\perp}$, for the Cluster s/c 1 crossing of an auroral boundary. The two curves labeled $22^{\circ}$ and $90^{\circ}$ in the upper panel correspond to two different angles of incidence for the upward ion beams.

upper panel correspond to two different incident angles for the upward ion beams collected by the ion detectors. The bottom panel shows the perpendicular potential, derived by integrating the perpendicular electric field along the spacecraft trajectory. The peak value of the perpendicular potential drop, indicated by the cross, is shown also in the upper panel for comparison. The peak potential can be seen to lie roughly in between the two parallel potential estimates. Although the location and magnitude of the parallel potential estimates are roughly in agreement, there are clear differences between the two curves. The calculated $\Delta \Phi_{\perp}$ variation reveals several small-scale adjacent potential drops, and the

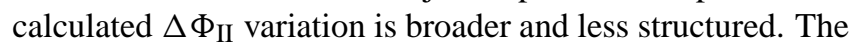
differences are likely due to a combination of the time mismatch between the two estimates (of the order of minutes) and temporal variations of the acceleration process.

Preliminary results for eight Cluster events, all of which are of quasi-static nature (used here to describe structures which are stable on time scales much longer than the electron transition time through the acceleration region and also longer than the satellite traversal time across the structure), are given in Table 1. The last three rows present the integrated perpendicular electric field, $\Delta \Phi_{\perp}$, the parallel potential drop, $\Delta \Phi_{\mathrm{II}}$, inferred from the electron data, and
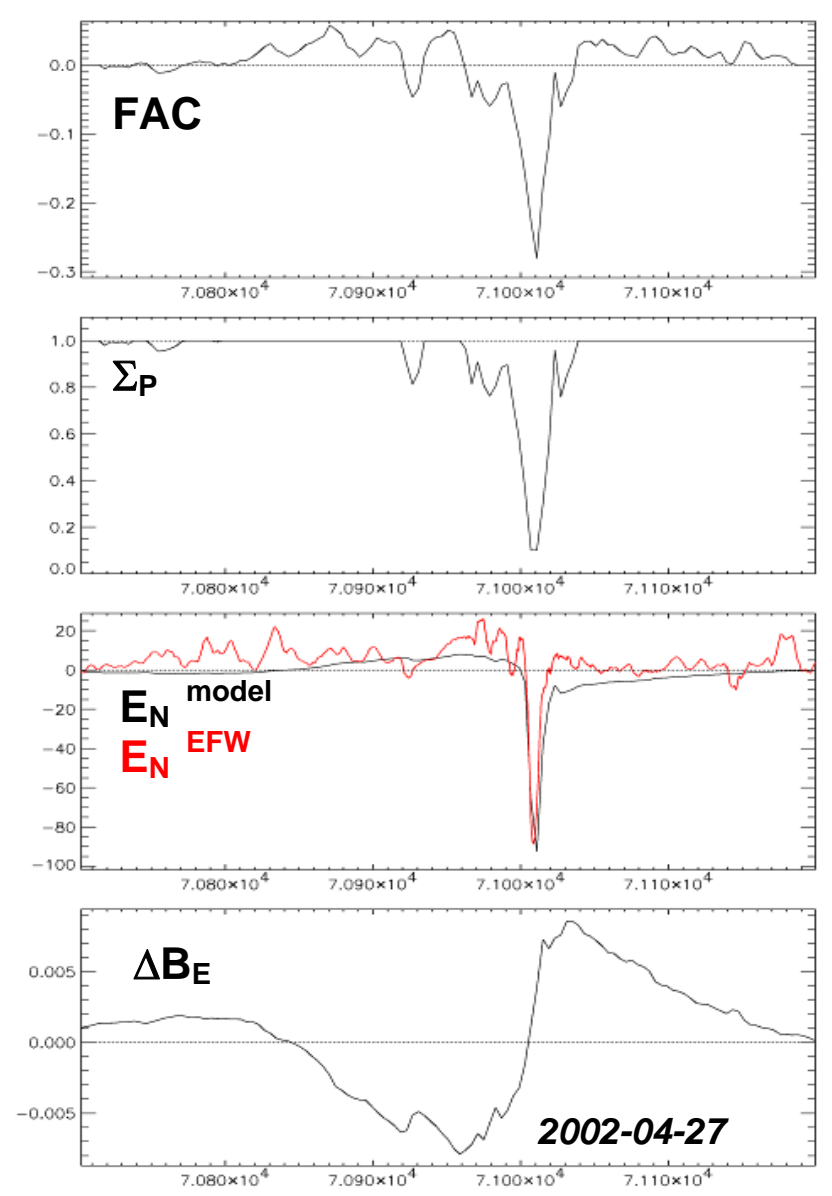

Fig. 6. Illustration of a case with a good correlation between the electric field and the downward FAC. The figure shows from top to bottom, the measured field-aligned current FAC, the calculated height-integrated Pedersen conductivity, the model and measured northward electric field component, and eastward residual magnetic field (reproduced from Karlsson et al., 2007). The good agreement between the model and Cluster results implies that the electric field maps perfectly between the ionosphere and Cluster altitude.

the ratio between the parallel and perpendicular potential, $k=\Delta \Phi_{I I} / \Delta \Phi_{\perp}$. It should be mentioned that the energy spectra of ions and electrons were inspected manually, which implies that the estimates of the potentials and coupling parameter are relatively rough. Note that for the five downward current events where the $k$-ratio could be estimated, it is one or close to one, in four of the five events. Although the events are too few to allow any firm conclusions, the results suggest that small-scale structures in the downward current region are mainly decoupled from the ionosphere, similar to smallscale structures in the upward current region.

An exception to the results presented in Table 1, that smallscale structures are typically decoupled, has been found for a special kind of downward current events shown in Fig. 6. The figure shows from top to bottom, the measured field-aligned 
Table 1. A summary of the characteristics of electric fields and field-aligned currents for 8 Cluster events, the first six of which are from downward current regions. The last three rows show the perpendicular potential, calculated from integrating the perpendicular electric field along the satellite orbit, the parallel potential, inferred from the characteristic energies of the upward electrons (events 1-6) and ions (events 7-8), and the coupling parameter $k$, defined as the ratio between the parallel potential and the perpendicular potential.

\begin{tabular}{|c|c|c|c|c|c|c|c|c|}
\hline Event date & $200101-14$ & $200102-14$ & $200204-27$ & $200205-19$ & $200211-25$ & $200302-28$ & $200305-01$ & $200305-03$ \\
\hline MLT & 03:30 & 00:20 & $20: 00$ & 20:00 & 05:30 & 00:00 & $20: 00$ & $23: 30$ \\
\hline Hem & NH & SH & $\mathrm{SH}$ & SH & $\mathrm{NH}$ & $\mathrm{SH}$ & SH & SH \\
\hline Potential & $\mathrm{U}$ & $\mathrm{U}$ & S & $S$ & $\mathrm{U}$ & S & $\mathrm{U}(1) \mathrm{S}(2)$ & $\mathrm{U}$ \\
\hline FAC up/down & down & down & down & down & down & down & up & up \\
\hline E-field peak (V/m) & 0.35 & 1.5 & 1.7 & 0.45 & 0.03 & 0.07 & 0.82 & $0.05-0.1$ \\
\hline Scale $(\mathrm{km})$ & 15 & $8(2)$ & $\begin{array}{l}1.5(2,4) \\
1.0(3)\end{array}$ & $2(1)$ & $1.6(1)$ & $4.7(1)$ & $\begin{array}{l}3.0(1,2,3) \\
3.2(4)\end{array}$ & $\begin{array}{l}10(2,4) \\
5(1)\end{array}$ \\
\hline $\mathrm{s} / \mathrm{c}$ & $1,2,3$ & $1,2,3,4$ & $2,3,4$ & $1,2,3,4$ & 1 & 1 & $1,2,3,4$ & $1,2,4$ \\
\hline$\Delta \Phi_{\perp}(\mathrm{kv})$ & 2 & 3 & 3 & $0.2-0.3$ & 0.6 & 12 & $\begin{array}{l}10 \\
12(4)\end{array}$ & $8(1)$ \\
\hline$\Delta \Phi_{\mathrm{II}}(\mathrm{kv})$ & 2 & No data & 3 & $0.2-0.3$ & 0.5 & $2-6$ & 10 & $\begin{array}{l}8(1) \\
12(4)\end{array}$ \\
\hline$K=\Delta \Phi_{\mathrm{II}} / \Delta \Phi_{\perp}$ & 1 & $?$ & 1 & 1 & 0.9 & $0.2-0.5$ & 1 & 1 \\
\hline
\end{tabular}

current FAC, the calculated height-integrated Pedersen conductivity, the model and measured northward electric field component, and eastward residual magnetic field (reproduced from Karlsson et al., 2007). Note the good agreement between the model and measured northward component of the electric field. For narrow current sheets, embedded in a large-scale system of upward and downward FAC closing in the ionosphere, current continuity requires that the electric field must intensify in the downward current region, because of the conductivity hole caused by up-flowing electrons and perpendicular transport of ions.

\section{Summary and conclusions}

An approach to study the quasi-static coupling of high-and low altitude electric fields have been outlined here and applied to a limited set of Cluster events. The results of this preliminary study suggest that small-scale structures in the downward current region are typically decoupled from the ionosphere, similar to small-scale structures in the upward current region. An exception to this is a special kind of downward current events, where the electric field correlates with the downward current and maps perfectly between the ionosphere and Cluster altitude. Recent statistical studies of the coupling issue, based on FAST data, show that a large majority of the events were neither completely coupled nor completely decoupled, with a clear difference between sheet-like and curved structures, the latter being more decoupled than the former. The coupling depends on the nature of the highly non-linear current voltage relation. Although this problem has been addressed in many recent papers, the current-voltage relationship is still a matter of debate in contrast to the well-established Knight (1973) relationship for the upward current region. A complication for the downward current region is that the depletion process limits the time for which the charge carriers are available in the original current channel. A way to handle this for the downward current sheet is to broaden, as has been observed in Cluster event studies as well as in numerical simulations of the downward current channel.

Acknowledgements. The author is grateful to a large number of people who have contributed to the success off the Freja and Cluster missions. The Freja project was supported by the Swedish National Space Board and by the German Ministry for Research and Technology through its project office at DLR. It was managed and operated by the Swedish Space Corporation under contract from the Swedish National Space Board. The Cluster project was supported by the European Space Agency and NASA. This study has been supported by the Swedish National Space Board and the Alfvén Laboratory Centre for Space and Fusion Plasma Physics, KTH, Stockholm.

Edited by: R. Pottelette

Reviewed by: R. Lundin and J. Jasperse 


\section{References}

Aikio, A. T., Mursula, K., Buchert, S., Forme, F., Amm, O., Marklund, G., Dunlop, M., Fontaine, D., Vaivads, A., and Fazakerley, A.: Temporal Evolution of Two Auroral Arcs as Measured by the Cluster Satellite and Coordinated Ground-Based Instruments, Ann. Geophys., 22, 4089-4101, 2004, http://www.ann-geophys.net/22/4089/2004/.

Balogh, A., Dunlop, M., Cowley, S., Southwood, D., Thomlinsson, J., Glassmeier, K., Musmann, G., Luhr, H., Buchert, S., Acuna, M., Fairfield, D., Slavin, J., Riedel, W., Schwingenschuh, K., and Kievelson, M.: The Cluster magnetic investigation, Space Sci. Rev., 79/1-2, 65-91, 1997.

Carlson, C. W., McFadden, J. P., Ergun, R. E., Temerin, M., Peria, W., Mozer, F. S., Klumpar, D. M., Shelley, E. G., Peterson, W. K., Moebius, E., Elphic, R., Strangeway, R. E., Cattell, C. A., and Pfaff, R.: FAST observations in the downward auroral current region: Energetic upgoing electron beams, parallel potential drops, and ion heating, Geophys. Res. Lett., 25, 2017-2020, 1998.

Elphic, R. C., Bonnell, J. W., Strangeway, R. J., Kepko, L., Ergun, R. E., McFadden, J. P., Carlson, C. W., Peria, W. J., Cattell, C. A., Klumpar, D., Shelley, E., Peterson, W. K., Moebius, E., Kistler, L., and Pfaff, R.: The auroral current circuit and field-aligned currents observed by FAST, Geophys. Res. Lett., 25, 2033-2036, 1998.

Ergun, R. E., Carlson, C. W., McFadden, J. P., Mozer, F. S., Delory, G. T., Peria, W. J., Chaston, C. C., Temerin, M., Elphic, R., Strangeway, R. J., Pfaff, R., Cattell, C. A., Klumpar, D., Shelly, E., Peterson, W. K., Moebius, E., and Kistler, L.: FAST satellite observations of electric field structures in the auroral zone, Geophys. Res. Lett., 25, 2025-2028, 1998.

Ergun, R. E., Carlson, C. W., McFadden, J. P., Strangeway, R. J., Goldman, M. V., and Newman, D. L.: Electron Phase-Space Holes and the VLF Saucer Source Region, Geophys. Res. Lett., 28, 3805-3808, 2001.

Gustavsson, G., Boström, R., Holback, B., Holmgren, G., Lundgren, A., Stasiewicz, K., Åhlén, L., Mozer, F., Pankow, D., Harvey, P., Berg, P., Ulrich, R., Pedersen, A., Schmidt, R., Butler, A., Fransen, A., Klinge, D., Thomsen, M., Fälthammar, C.-G., Lindqvist, P. -A., Christenson, S., Holtet, J., Lybekk, B., Stein, T., Tanskanen, P., Lappalainen, K., and Wygant, J.: The Electric Field and Wave Experiment for the Cluster mission, Space Sci. Rev., 79/1-2, 137-156, 1997.

Hwang, K.-J., Lynch, K. A., Carlson, C. W., Bonnell, J. W., and Peria, W. J.: Fast Auroral Snapshot observations of perpendicular DC electric field structures in downward auroral current regions: Morphology, J. Geophys. Res., 111, A09205, doi:10.1029/2005JA011471, 2006a.

Hwang, K.-J., Lynch, K. A., Carlson, C. W., Bonnell, J. W., and Peria, W. J.: Fast Auroral Snapshot observations of perpendicular DC electric field structures in downward current regions: Implications, J. Geophys. Res., 111, A09206, doi:10.1029/2005JA011472, 2006 b.

Jasperse, J. R.: Ion heating, electron acceleration, and the selfconsistent E-field in downward auroral current regions, Geophys. Res. Lett., 25, 3485-3488, 1998.

Jasperse, J. R., Basu, B., Lund, E. J., and Bouhram, M.: Gyrotropic guiding-center fluid theory for turbulent inhomogeneous magnetized plasma, Phys. Plasmas, 13, 072903, doi:10.1063/1.2220006, 2006.
Jasperse, J. R., Basu, B., Lund, E. J., and Bouhram, M.: Gyrotropic guiding-center fluid theory for the turbulent heating of magnetospheric ions in the downward Birkeland current regions II, Phys. Plasmas, 13, 112902, doi:10.1063/1.2364475, 2006.

Johnstone, A., Alsop, C., Burge, S., Carter, P. J., Coates, A. J., Coker, A. J., Fazakerley, A, Grande, M., Gowen, R. A., Gurgiolo, C., Hancock, B. K., Narheim, B., Preece, A., Sheather, P. H., Winningham, J., and Woodcliffe, R. D., PEACE: A plasma electron and current experiment, Space Sci. Rev., 19/1-2, 351-398, 1997.

Karlsson, T. and Marklund, G.: Simulations of effects of smallscale auroral current closure in the return current region, Physics of space plasmas, 15, 401-406, 1998.

Karlsson, T., Marklund, G. T., Figueiredo, S., Johansson, T., and Buchert, S.: Separating Spatial and Temporal Variations in Auroral Electric and Magnetic Fields by Cluster Multipoint Measurements, Ann. Geophys., 22, 2463-2472, 2004, http://www.ann-geophys.net/22/2463/2004/.

Karlsson, T., Brenning, N., Marghitu, O., Marklund, G., and Buchert, S.: High-altitude signatures of ionospheric density depletions caused by field-aligned currents, arXiv:0704.1610v1 [physics.space-ph], 2007.

Knight, S.: Parallel electric fields, Planet. Space Sci., 21, 741, 1973

Lynch, K. A., Bonnell, J. W., Carlson, C. W., and Peria, W. J.: Return current region aurora: E-parallel, j(z), particle energization, and broadband ELF wave activity, J. Geophys. Res., 107(A7), 1115, doi:10.1029/2001JA00134, 2002.

Marklund G. T., Blomberg, L. G., Fälthammar, C.-G., and Lindqvist, P.-A.: On Intense Diverging Electric Fields Associated with Black Aurora, Geophys. Res. Lett., 21, 1859-1862, 1994.

Marklund, G., Karlsson, T., and Clemmons, J.: On low-altitude particle acceleration and intense electric fields and their relation to black aurora, J. Geophs. Res., 102, 17 509-17 522, 1997.

Marklund, G., Ivchenko, N., Karlsson, T., Fazakerley, A., Dunlop, M., Lindquist, P.-A., Buchert, S., Owen, 390 C., Taylor, M., Vaivalds, A., Carter, P., André, M., and Balogh, A.: Temporal evolution of the electric field accelerating electrons away from the auroral ionosphere, Nature, 414, 724-727, 2001.

Marklund, G. T., Karlsson, T., Figueiredo, S., Johansson, T., Lindqvist, P.-A., André, M., Buchert, S., Kistler, L. M., and Fazakerley, A.: Characteristics of quasi-static potential structures observed in the auroral return current region by Cluster, Nonlin. Processes Geophys., 11, 709-720, 2004, http://www.nonlin-processes-geophys.net/11/709/2004/.

Marklund, G., André, M., Lundin, R., and Grahn, S.: The Swedish Small Satellite Program for Space Plasma Investigations, Space Sci. Rev., 111, 377-413, 2004.

Marklund, G. T., Karlsson, T., Figueiredo, S., Johansson, T., Lindqvist, P.-A., André, M., Buchert, S., and Kistler, L.: Dynamics and characteristics of electric-field structures in the auroral return current region observed by Cluster, Physica Scripta, 34-43, 2006.

Paschmann, G., Haaland, S., and Treumann, R., Auroral plasma physics, Space Sci. Rev. 103, 1-4, 2002.

Réme, H., Bosqued, J. M., Sauvaud, J. A., Cros, A., Dandouras, J., Aoustin, C., Bouyssou, J., Camus, T., Cuvilo, J., Martz, C., Médale, J. L., Perrier, H., Romefort, D., Rouzaud, J., D’Uston, C.,M“obius, E., Crocker, K., Granoff, M., Kistler, L. 
M., Popecki, M., Hovestadt, D., Klecker, B., Paschmann, G., Scholer, M., 400 Carlson, C. W., Curtis, D. W., Lin, R. P., Mcfadden, J. P., Formisano, V., Amata, E., Bavassano-Cattaneo, M. B., Baldetti, P., Belluci, G., Bruno, R., Chionchio, G., Lellis, A. D., Shelley, E. G., Ghielmetti, A. G., Lennartsson,W., Korth, A., Rosenbauer, H., Lundin, R., Olsen, S., Parks, G. K., Mccarthy, M., and Balsiger, H.: The Cluster ion spectrometry (CIS) experiment, Space Sci. Rev., 19/1-2, 303-350, 1997.

Streltsov, A. V. and Marklund, G. T.: Divergent electric fields in downward current channels, J. Geophys. Res., 111, A07204, doi:10.1029/2005JA011196, 2006.
Temerin, M. and Carlson, C. W.: Current-Voltage relations in the downward auroral current region, Geophys. Res. Lett., 25, 23652368, 1998.

Vedin, J. and Rönnmark, K.: Electrostatic potentials in the downward auroral current region. J. Geophs. Res., 110, A08207, doi:10.1029/2005JA011083, 2005.

Weimer, D. R., Goertz, C. K., and Gurnett, D.: Auroral zone electric fields from DE 1 and DE 2 at magnetic conjunctions, J. Geophys. Res., 90, 7479-7494, 1985. 